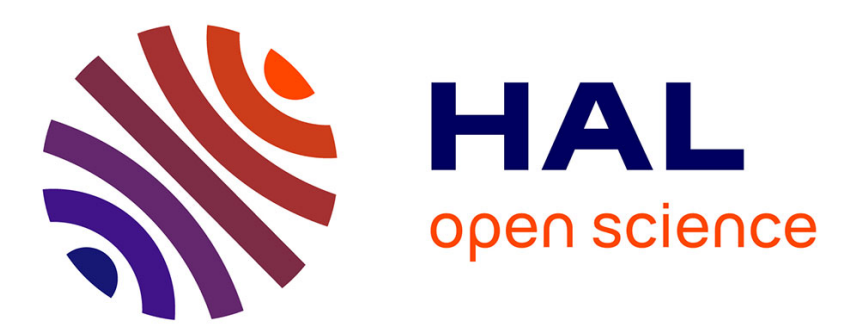

\title{
Acoustics of porous materials with partially opened porosity
}

Philippe Leclaire, Thomas Dupont, Raymond Panneton

\section{To cite this version:}

Philippe Leclaire, Thomas Dupont, Raymond Panneton. Acoustics of porous materials with partially opened porosity. Journal of the Acoustical Society of America, 2013, 134 (6), pp.4630-4641. 10.1121/1.4824836 . hal-01331511

\section{HAL Id: hal-01331511 \\ https://hal.science/hal-01331511}

Submitted on 13 Jun 2016

HAL is a multi-disciplinary open access archive for the deposit and dissemination of scientific research documents, whether they are published or not. The documents may come from teaching and research institutions in France or abroad, or from public or private research centers.
L'archive ouverte pluridisciplinaire HAL, est destinée au dépôt et à la diffusion de documents scientifiques de niveau recherche, publiés ou non, émanant des établissements d'enseignement et de recherche français ou étrangers, des laboratoires publics ou privés. 


\title{
Acoustics of porous materials with partially opened porosity
}

\author{
P. Leclaire ${ }^{\text {a) }}$ and T. Dupont \\ DRIVE, Université de Bourgogne, 49 rue Mademoiselle Bourgeois, 58027 Nevers cedex, Bourgogne 58027, \\ France \\ R. Panneton \\ GAUS, Department of Mechanical Engineering, Université de Sherbrooke, Sherbrooke, Quebec, JIK 2RI, \\ Canada
}

\begin{abstract}
A theoretical and experimental study of the acoustic properties of porous materials containing dead-end (or partially opened) porosity was recently proposed by Dupont, Leclaire, Sicot, Gong, and Panneton [J. Appl. Phys. 110, 094903 (2011)]. The present article provides a description of partially opened porosity systems and their numerous potential applications in the general context of the study of porous materials, the classical models describing them, and the characterization techniques. It is shown that the dead-end pore effect can be treated independently and that the description of this effect can be associated with any acoustic model of porous media. Different theoretical developments describing the dead-end porosity effect are proposed. In particular, a model involving the average effective length of the dead-end pores is presented. It is also shown that if the dead-end effect can be treated separately, the transfer matrix method is particularly well suited for the description of single or multilayer systems with dead-end porosity.
\end{abstract}

\section{INTRODUCTION}

Fluid-saturated porous materials are of great importance in an increasing number of applications in the automotive and transportation systems industries, in civil engineering, or even in the energy management industry. Since they contain a fluid, these heterogeneous materials are intrinsically lighter than other more classical materials (homogeneous solids). They can also exhibit very interesting properties in different fields such as mechanics, vibration and acoustics, fluid mechanics, thermal physics, or even electromagnetism. For this reason, they can be considered as "multifunctional."

These materials can easily be obtained from recycling processes and can be produced from green materials such as wood shaving particles.

In the field of automobile and transportation systems, there is an increasing interest in the study of materials susceptible to absorb impacts and shocks in order to improve safety but also contributing to the noise reduction to improve the acoustic comfort

The microstructure of the studied materials is "complex" and furthermore, in vibroacoustic applications, the elastic moduli as well as the wavenumbers that describe the wave propagation in these materials, are mathematically complex as these moduli and constants have imaginary parts in order to account for vibration damping and sound absorption mechanisms. These materials will therefore be referred to as "complex materials" in this article. This article aims at studying and characterizing a certain class of these materials, namely the materials containing "partially opened pores" or pores for

\footnotetext{
a) Author to whom correspondence should be addressed. Electronic mail: Philippe.Leclaire@u-bourgogne.fr
}

which "one end is not connected to the surroundings." For these pores, we can also use the terms "dead-ends," "impasses," or "cul-de-sac." The presence of these peculiar pores may result in a significant modification of the acoustic indicators (absorption coefficient and transmission loss).

This article proposes to study, theoretically and numerically, the acoustic properties of these materials. An experimental verification on artificially created simplified samples with well-controlled microstructure or on complex materials used in acoustic engineering is also presented. The experimental verification of the model predictions on simplified materials with well-controlled microgeometry involving quarter-wavelength cavities and Helmholtz-type resonators brings about new technological challenges in the field of material manufacturing. It is thought that these challenges will be the same as those encountered for the design of acoustic metamaterials.

The introduction of partially opened porosity in the description of the acoustic properties of complex materials gives rise to new theoretical research and opens up new scopes in the field of material characterization methods. The theoretical and experimental challenges are numerous and the applications are wide range. For example, it is thought that materials obtained through recycling processes may contain a non-negligible proportion of dead-end porosity.

Dupont et al. ${ }^{1}$ recently proposed a theoretical and experimental description of the acoustic properties of porous materials containing dead-end pores. In the present article, a more general approach to this problem is proposed. In particular, it is shown that for a linear propagation, the partially opened porosity effect can be described in a model (that can be referred to as "acoustic model of partially opened porosity effect") and that this model can be developed independently from any classical acoustic model of porous material that does not 
account for partially opened porosity such as the Delany and Bazley model or the model by Johnson-Champoux-Allard, for example. The acoustics of porous materials containing dead-end pores can therefore be described by the combination of two elementary theoretical bricks: a classical acoustic model of porous media and a specific model of the dead-end porosity effect. Different possibilities for the latter are reviewed. A new model involving a new parameter: The average effective length of the dead-end pores $l_{\mathrm{DE}}^{e}$ is proposed. This parameter is an alternative parameter to the average length of the dead-ends $l_{\mathrm{DE}}$ proposed in Ref. 1. Although in the present article only theoretical and experimental results in the rigid frame approximation are presented, it is thought that the new parameter $l_{\mathrm{DE}}^{e}$ will play a role in the theoretical description of poroelastic materials with dead-end pores.

Section II of this article proposes a short review of the main acoustic models of fluid-saturated porous media to date and of their associated parameters. Section III is devoted to the methods for the characterization of the physical and mechanical properties of porous materials. In Sec. IV, the latest theoretical developments on porous materials incorporating partially opened porosity are proposed. Different models describing the partially opened pore effect are proposed. Experimental results are presented in Sec. V, where porous materials containing dead-end porosity and materials with well-controlled microstructural parameters are studied. In Sec. VI, recent experimental techniques developed for the measurement of the dead-end porosity are briefly presented.

\section{MODELS AND PARAMETERS}

\section{A. Different models of the wave propagation in fluid-saturated porous media}

Over the past 20 years, a number of models to calculate the acoustic behavior of porous materials have been developed. Although these models are based on physical sound theories, they require a number of material parameters and the output of a calculation will depend on the accuracy of the input parameters. Depending on the complexity of the porous material and the configuration to be modeled, up to seven physical parameters and two or more elastic parameters may be needed. A major reference in this area is the book by J. F. Allard ${ }^{2}$ (1st and 2 nd eds.), which provides the theoretical framework to solve a great number of engineering acoustics problems.

The interest for the sound propagation in porous materials goes back to the end of the 19th century with the work of Lord Rayleigh. Following the works by Kirchhoff ${ }^{3}$ and by Crandall, ${ }^{4}$ Zwikker and Kosten ${ }^{5}$ contributed substantially to the field in 1949 by offering a model of sound propagation in materials containing cylindrical pores and taking into account the viscous and thermal interaction between air and the solid. In 1956 Biot $^{6}$ published a refined model of the acoustic wave propagation in fluid saturated porous media accounting for elastic, inertial, and viscous couplings between the two phases. In this model, the medium is poroelastic and viscous frictions between the solid and the fluid are included. Biot studied the low and high frequency behaviors. In the high frequency regime, the fluid flow in the pore deviates from
Poiseuille's flow. The Biot model is self-consistent and selfsufficient at high as well as at low frequencies. Yet, the Biot theory remained relatively unrecognized and unappreciated until the years 1970-1980. From these years, this theory has been rediscovered and its success never diminished. The scientific community realized the great potential of Biot's theory in numerous fields such as geophysics, petroleum prospection, the automotive industry, or medicine. Following Biot's theory, very interesting and useful developments were proposed. The Johnson-Champoux-Allard model in acoustic engineering, as well as many other models developed recently, are based on this theory.

Over the years, other types of models were developed, some of which are empirical. For example, in 1970, Delany and Bazley ${ }^{7}$ proposed an empirical model describing the sound wave propagation in fibrous materials. This model is simple and has been very popular in engineering acoustics. Several extensions of the Delany and Bazley model were proposed. Other models are more refined and are based on other principles such as the Wilson model, ${ }^{8}$ which is based on relaxation processes.

This article is concerned with the most refined models derived from Biot's theory and capable, if needed, of accounting for poroelasticity. The attention is also focused on the parameters (microstructural, physical, and mechanical) associated with these models. Attenborough ${ }^{9}$ has shown the importance of tortuosity and of the parameters related to the complexity of the pore geometry at high frequencies. Johnson et al. ${ }^{10}$ have studied the high frequency asymptotic behavior of a Newtonian fluid subjected to a pressure gradient in the pores of a porous medium. They have introduced the concepts of dynamic tortuosity and of dynamic permeability. They studied the low and high frequency behaviors of these functions and proposed a function linking the two behaviors. Following the work by Johnson et al., Champoux and Allard ${ }^{11}$ studied the temperature gradient in the fluid between the thermal boundary layer and the inside of the pore away from the boundary layer at high frequency. The model referred to as the Johnson-Champoux-Allard (JCA) model involves five physical parameters including the viscous and thermal characteristic lengths. The parameters of the models are reviewed further down in this article. Lafarge et al. ${ }^{12}$ brought a further refinement to the JCA by modeling the thermal exchanges between the solid and the fluid at low frequencies. Therefore, their model involves six parameters. Pride et al. ${ }^{13}$ studied the low frequency inertial behavior of the equivalent fluid and proposed a correction of the low frequency limit of the real part of the dynamic tortuosity of Johnson et al. Horoshenkov et al. proposed a model involving a Padé approximation of the high frequency viscosity correction function and capable of accounting for the pore size distribution. ${ }^{14}$

The different models derived from Biot's theory (the models by Attenborough, Johnson et al., Champoux and Allard, Lafarge et al., Pride et al., Horoshenkov and Swift) are capable, if necessary, of including the poroelastic behavior of the solid frame. However, in the cases where the solid is limp and does not offer resistance to deformation or where the solid is much more rigid and heavier than the fluid, the porous medium can be considered as an "equivalent fluid" in 
the "limp frame" or "rigid frame" approximation, respectively (Panneton ${ }^{15}$ ). In this case, only one of the two Biot coupled equations is necessary and the acoustic model is simpler. These different models can be incorporated into numerical methods such as the finite element method for applications involving the vibrations and the acoustics of poroelastic materials. The numerical solution of specific problems involving the two Biot coupled equations of poroelasticity including the solid and the fluid displacements $(\boldsymbol{u}, \boldsymbol{U})$ can require a high computation time due to the great number of degrees of freedom. For this reason, Attala et al. ${ }^{16}$ proposed a new formulation of Biot's coupled equations, in which the conjugate variables $(\boldsymbol{u}, \boldsymbol{U})$ are replaced by $(\boldsymbol{u}, p)$, where $p$ represents the fluid pressure. Since $p$ is a scalar, this formulation allows, without any concession on the generality, to reduce the number of degrees of freedom and therefore, the computation time.

Accompanying the alternatives to the Biot theory and improvements described in the previous sections, useful extensions of Biot's theory describing new phenomena were proposed over the years among which we find: the high frequency limit of the Biot-based models and the transition with the scattering theories (Leclaire et al. ${ }^{17}$ ), the effect of double scale porosity (Olny and Boutin ${ }^{18}$ ), a time domain approach of the wave propagation (Fellah and Depollier ${ }^{19}$ ), the study of inhomogeneous rigid frame porous materials (De Rijck et al. ${ }^{20}$ ), poroelastic inhomogeneous porous materials (Gautier et al. $^{21}$ ), bottom-up approaches (Chevillote et $a l^{22}$ ), or partially opened porosity materials (Leclaire et al., ${ }^{23}$ confirmed and improved by Dupont et al. $\left.{ }^{1}\right)$.

\section{B. The parameters of the models}

The porosity $\phi$ was the first parameter defined in early models. From Darcy's law of fluid flow through porous media, the notion of permeability $\mathrm{k}_{0}$ was then introduced in the acoustics of porous media to account for viscous frictions. In his original work of 1956 in the high frequency regime, Biot $^{6}$ introduced the concepts of tortuosity (sinuosity factor $\xi$ ) and also the structural factor $\delta$ (deviation from cylinders with constant circular cross section) characterizing the complexity of the pore geometry at high frequencies. Attenborough ${ }^{9}$ offered an alternative definition for the tortuosity $(q)$ and proposed a description in terms of static and dynamic shape factors. Johnson et al. ${ }^{10}$ introduced the concepts of dynamic tortuosity (with its high frequency limit $\alpha_{\infty}$ ), of dynamic permeability and of viscous characteristic length $\Lambda$. This parameter is related to the pore microgeometry. The dynamic compressibility and thermal characteristic lengths $\Lambda^{\prime}$ were then introduced by Champoux and Allard. ${ }^{11}$ Lafarge et al. ${ }^{12}$ introduced the thermal permeability $\mathrm{k}_{0}^{\prime}$. Pride et al. ${ }^{13}$ studied the low frequency limit of the tortuosity.

\section{CHARACTERIZATION OF POROUS ACOUSTIC MATERIALS}

\section{A. Physical parameters}

Two kinds of methods can be considered for the measurement of porosity and other parameters: the non-acoustic methods and the acoustic methods. For porosity, a nonacoustic method is based on Boyle's law for isothermal processes $\left(\right.$ Beranek $\left.^{24}\right)$, the principle of which has become a standard [ASTM D2856-949 (Ref. 25)]. An improved and modernized version was proposed by Champoux et al. ${ }^{26}$ Leclaire et $a{ }^{27}{ }^{27}$ used an improved version of Beranek's setup to compare air volumes. Other well-known classical methods are based on density measurements. Inspired from this principle, Salissou and Panneton ${ }^{28}$ proposed a method based on pressure/mass measurements. Panneton and Gros ${ }^{29}$ presented a method based on a missing mass.

For the flow resistivity or the permeability (the flow resistivity being inversely proportional to the permeability), standard methods are based on the pressure gradient and flow velocity measurements. ${ }^{30}$

Other well-known original non-acoustic methods include the Brunauer-Emmet-Teller measurement ${ }^{31}$ for the evaluation of $\Lambda^{\prime}$ and electrical conductivity measurements for $\alpha_{\infty}$ [this technique can be attributed to geophysicists (see Ref. 32) and involves an electrical formation factor known to Lord Rayleigh].

Acoustic methods are based on the inversion of physical parameters from acoustic measurement data. Depending on the known parameters in the problem considered, the appropriate parameters can be deduced. Different versions of acoustic methods were used in the past to determine the porosity, the tortuosity, the permeability or the flow resistivity, or even the viscous and thermal characteristic lengths. In addition, low frequency methods (impedance tube measurements) and high frequency methods (ultrasonic measurement) can be distinguished. In the low frequency methods (for example Ref. 33), the impedance and transmission tube measurements with inverse or indirect methods can be used.

Based on the pioneering experiments by Nagy, ${ }^{34}$ original high frequency methods (for example, Refs. 35 and 36) involving the use of ultrasonic waves in air-saturated materials were developed, in particular in the Laboratorium voor Akoestiek en Thermische Fysica (ATF) of the Katholieke Universiteit Leuven in Belgium and in the Laboratoire d'Acoustique de l'Université du Maine (LAUM) in France. Allard et $a l^{35}$ were the first to measure the tortuosity of highly porous polyurethane foams saturated by air. Leclaire et $a l .{ }^{36}$ improved this method to determine the tortuosity but also the viscous and thermal characteristic lengths. Several extensions were proposed since these first experiments. Fellah et $a l .^{37}$ proposed, in the time domain, a method for measuring the porosity and tortuosity based on the solution of inverse problem using the reflected waves by the first interface of porous material.

\section{B. Elastic and viscoelastic parameters}

Approximating the porous material as an equivalent fluid (in the rigid or limp limits) gives good results in many applications for many sound-absorbing materials. However, this approximation does not always hold, and in a number of cases, the acoustic material has to be described with the full theory for sound propagation in poroelastic media. To accompany Biot's theory, the elastic moduli of poroelastic materials 
saturated by fluids were defined by Biot and Willis ${ }^{38}$ with the use of the concept of gedanken experiments in 1957. The elastic coefficients of the "dry" (unsaturated) frame are needed in the models. This leads to two options: either designing an experiment in vacuum, where the numerical inversion to extract the elastic coefficients will be easy, or designing an experiment in air that is easier to perform but where the numerical inversion may require the full model. Pioneering research on this subject was developed by Pritz. ${ }^{39}$ Moreover, many sound-absorbing or sound-damping materials are viscoelastic and their elastic coefficients may depend on frequency and temperature. This subject is relatively unexplored and represents a challenging research field.

\section{Low frequency methods}

The simplest experiments to evaluate the elastic coefficients are based on the measurement of a vibrational resonance frequency in a sample (rod-shaped, bar-shaped, or a plate) of small dimensions compared to the wavelength involved. ${ }^{40}$ These methods are based on the vibration of a sample of finite size resulting in a transfer function with resonance peaks. The effect of resonances in the transfer function is that the moduli are determined with a precision that varies depending on the frequency area examined. The precision is best around the resonance frequencies and poorer elsewhere. In addition, in the experiment for measuring Young's modulus, the wavelengths involved are greater than or in the order of the length of the rod, they must be greater than the lateral size and the method is limited to low frequencies (typically $400 \mathrm{~Hz}$ ). Since most of the porous materials are also designed to attenuate structureborne sound, their structural damping is high, and as a consequence, the elastic coefficients are frequency dependent. Evaluating the elastic coefficients at low frequencies can have a poor predictive value for higher frequencies.

\section{Higher frequency methods}

Higher frequency methods for measuring the shear modulus based on the structure-borne Rayleigh wave propagation at the interface between a porous layer and the surrounding fluid $^{41}$ were proposed. The Rayleigh wave method allowed measurements up to $3 \mathrm{kHz}$. Other methods based on the propagation on guided and interface waves were also proposed more recently by Boeckx et al ${ }^{42}$ Together with a complete description of a soft porous layer lying on a rigid substrate or in the Lamb boundary conditions, they proposed a novel experimental technique for measuring the phase velocities of all the possible modes. The method is based on the generation and detection of standing waves in the layer and on the spatial Fourier transform of the standing-wave pattern. Research in this area and on the influence of temperature in a wide frequency range is promising and ongoing.

\section{MODEL FOR THE ACOUSTIC PROPAGATION IN POROUS MATERIALS WITH PARTIALLY OPENED POROSITY}

Recent research on the acoustic properties of porous materials ${ }^{1,22,23}$ has revealed the possible existence of pores

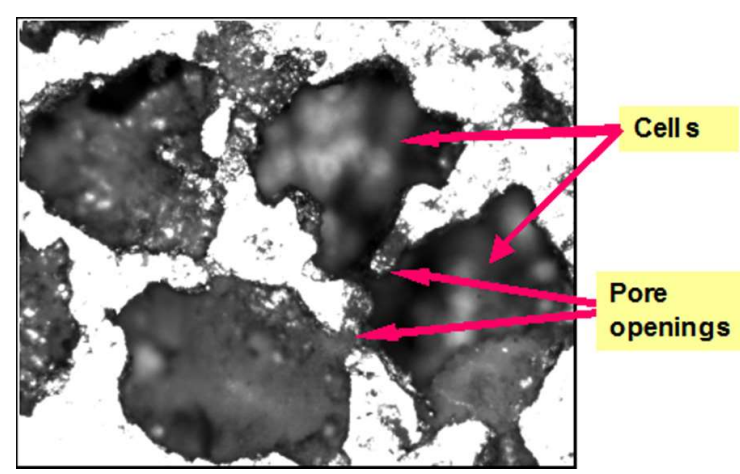

FIG. 1. (Color online) Example of porous material containing dead-end porosity (porous aluminum) (Ref. 1). The dark regions correspond to the pore space.

that are partially opened (pores opened at one end only or dead-end pores). It is thought that many complex materials such as porous metallic foams, certain recycled materials, ${ }^{43}$ asphalt, porous rocks or even bones can exhibit these features. For these materials, one of the Biot assumptions: the one on the flow of a fluid inside a pore may not be fulfilled. Although dead-end porosity is not new and has already been reported by geophysicists, no model was able until now to describe precisely its influence on the acoustic properties of porous materials. In the model proposed by Dupont et al., two new physical parameters were introduced: namely, the average length of the dead-ends $l_{\mathrm{DE}}$ and the dead-end porosity $\phi_{\mathrm{DE}}$. Figure 1 shows an example of porous material containing dead-end porosity. In this example, the fact that some pores may be connected to the surroundings by only one end is responsible for the absence of fluid flow in all the pores of the sample during the wave propagation. Figure 2 shows the relationships between the different porosities in a complex porous material incorporating dead-end porosity. The dead-end porosity and the average length of the deadends are discussed more quantitatively in the next sections.

\section{A. Microscopic description of the dead-end pores}

The material of Fig. 1 can be modeled at the microscopic scale with the help of a simple model. A duct of constant cross section $\mathrm{S}$ is considered in Fig. 3. The right end of the duct is connected to two auxiliary ducts 1 and 2 of constant section $S_{1}$ and $S_{2}$, respectively.

For the Y-shape junction of Fig. 3, acoustic admittances $\mathrm{Y}, \mathrm{Y}_{1}$, and $\mathrm{Y}_{2}$ can be defined for each branch. These are

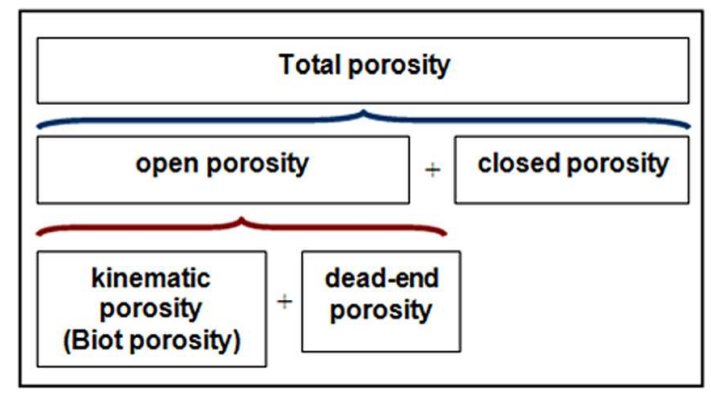

FIG. 2. (Color online) Relationships between the different porosities in a porous complex material. 


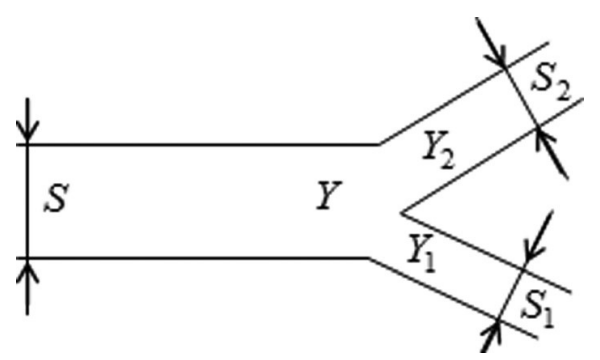

FIG. 3. Y-shape junction between three branches in a porous medium.

related to the characteristic impedance of each branch through the following relations: ${ }^{44}$

$$
Y=\frac{S}{Z}, Y_{1}=\frac{S_{1}}{Z_{1}}, Y_{2}=\frac{S_{2}}{Z_{2}}
$$

Since the boundary conditions are that the pressure must be the same on both sides of the junction and that the volume velocity into the junction must equal that out; and assuming a left to right propagation, the relationship between the acoustic admittances is ${ }^{44}$

$$
Y=Y_{1}+Y_{2} \text {. }
$$

If we now consider that one of the branches-branch 1 , for example-is closed (see Fig. 4) the previous relation (2) remains valid with the difference that $Y$ now represents a local admittance at the end of the main branch while $Y_{1}$ corresponds to the local admittance at the entrance of branch 1 . If branch 1 has a constant cross section and if the closing wall is rigid and perpendicular to the branch axis, the admittance $Y_{l}$ is given by

$$
Y_{1}=\frac{S_{1}}{-j Z_{C} \operatorname{cotan}(k l)}
$$

where $Z_{C}$ is the characteristic impedance of the fluid, $k$ is the wavenumber, $l$ is the length of branch 1 , and $j$ is the unit imaginary complex number [a time dependence in $\exp (j \omega t)$ has been chosen, $\omega$ being the angular frequency].

\section{B. Macroscopic description of the dead-end pores at the laboratory scale}

In most of the cases in audible acoustics, the wavelengths are much greater than the characteristic sizes of the heterogeneities and the propagation is in the linear regime.

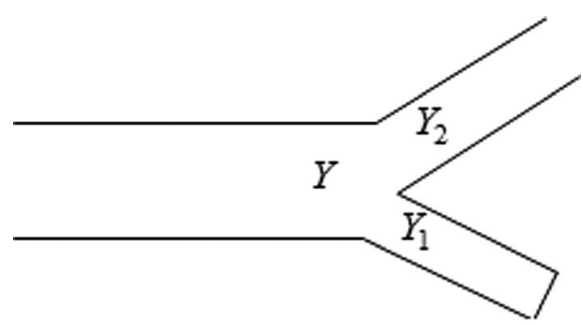

FIG. 4. Y-shape junction in a porous medium with one branch closed.
The behavior described at the microscopic scale can then be homogenized using a simple relationship:

$$
\bar{Y}=\bar{Y}_{1}+\bar{Y}_{2},
$$

where $\bar{Y}, \bar{Y}_{1}$, and $\bar{Y}_{2}$ represent the averaged quantities associated with $Y, Y_{1}$, and $Y_{2}$, respectively, in a homogenization volume in the porous medium. It is thought that this relationship (proposed without demonstration) and without crossterms is valid in many cases for linear propagation. It will be seen later that this equation seems to provide good results for our experimental study. In addition, it is assumed that in the materials studied, the cross sections of all branches are statistically uniform so that they do not play a role in Eq. (4). This last equation can easily be interpreted. For a linear propagation, the acoustic behavior of a material containing dead-end pores is given by the simple sum of the two contributions corresponding to the kinematic porosity (also called the Biot porosity in the present article) $\phi_{B}$ and to the deadend porosity $\phi_{\mathrm{DE}}$. These two porosities are related to the total open porosity $\phi$ through the relation (see Fig. 1):

$$
\phi=\phi_{B}+\phi_{\mathrm{DE}}
$$

The kinematic porosity corresponds to the pores where all Biot's assumptions are fulfilled. In his study of 1956 on the acoustic wave propagation (low and high frequency regimes) in a fluid-saturated porous medium, Biot made the assumption that the flow of fluid associated with the passage of the wave was taking place within all the pores of the medium. While this assumption is valid for many porous materials, it is thought that it is not in materials with dead-end pores. Since the dead-end pores are partly open (they are closed at one end), no flow occurs inside them. As a consequence, a fraction of the pores in the material, the dead-ends, do not obey Biot's assumption on fluid flow and do not participate in the overall attenuation by viscous frictions. The dead-end porosity can be due to the presence along the open pores of quarterwavelength cavities or Helmholtz-type resonators. Zwikker and Kosten ${ }^{5}$ studied the acoustic properties of air-saturated perforated materials and noticed the effect of the presence of lateral cavities along the perforations, but they did not offer a theoretical description for this phenomenon. They stated that "notwithstanding the application of a pressure gradient, the air in the lateral cavity remains largely at rest," and that "the pressure-gradient only succeeds in accelerating the air in the main pore, leaving the air in the side hole at rest." In their description, the lateral cavities were considered as acoustically "inert" and only a porosity correction was proposed to account for them. In the present study however, it is thought that the dead-end pores act as quarter wavelength cavities and as Helmhotz-type resonators, resulting in possible resonance peaks in the acoustic indicators curves. These resonances would correspond to the presence of standing wavefields with no fluid flow but solely acoustic pressure variations. In the present model, these variations are taken into account through the term $\bar{Y}_{2}$. Also, the influence of the dead-end pores on the physical parameters (tortuosity, flow resistivity, characteristic lengths, ...) is discussed later in this article. 


\section{Insertion of the dead-end porosity effect within any classical acoustic model}

The dead-end porosity effect can be added to any acoustic model of porous material (or any model of perforated materials) through the following procedure:

(i) the contribution $\bar{Y}_{2}$ in Eq. (4) is calculated first using an acoustic model in which $\phi_{B}$ is considered. In Ref. 1, the classical JCA model was used;

(ii) the dead-end contribution $\bar{Y}_{1}$ is then calculated using a model in which $\phi_{\mathrm{DE}}$ is considered. The relationship between the porosities is given by Eq. (5). Examples of possible models involving $\phi_{\mathrm{DE}}$ and the average length of the dead-end $l_{\mathrm{DE}}$ or another length $l_{\mathrm{DE}}^{e}$ are proposed in the next section.

The acoustic indicators are then deduced from the admittance $\bar{Y}$ of the material with dead-end pores given by Eq. (4). The procedure is illustrated with the help of Fig. 5.

\section{Simple models for the acoustic fields within the dead-end pores}

\section{Cavities involving the dead-end porosity $\phi_{\mathrm{DE}}$ and the average length of the dead-end pores $I_{\mathrm{DE}}$}

The problem consists now in finding a suitable expression for the admittance $\bar{Y}_{1}$. If we consider that all the deadend pores constitute a collection (in a sufficient number) of quarter wavelength cavities, we can define from Eq. (3) an average value, integrated over a homogenization volume $V_{\mathrm{DE}}$ of dead-end pores:

$$
\bar{Y}_{1}=\frac{j}{Z_{C}} \frac{\iiint_{V_{\mathrm{DE}}} \tan (k l) d V}{\iiint_{V_{\mathrm{DE}}} \mathrm{d} V},
$$

where $Z_{C}$ is the characteristic impedance of the fluid in the cavity of length $l$ and $k$ the wavenumber. It may be noticed that Eq. (3) is obtained from the study of the pressure and particle velocity fields in an open-closed duct with constant cross section. It is known that in such a configuration, a standing wavefield is obtained. $\bar{Y}_{1}$ then represents the

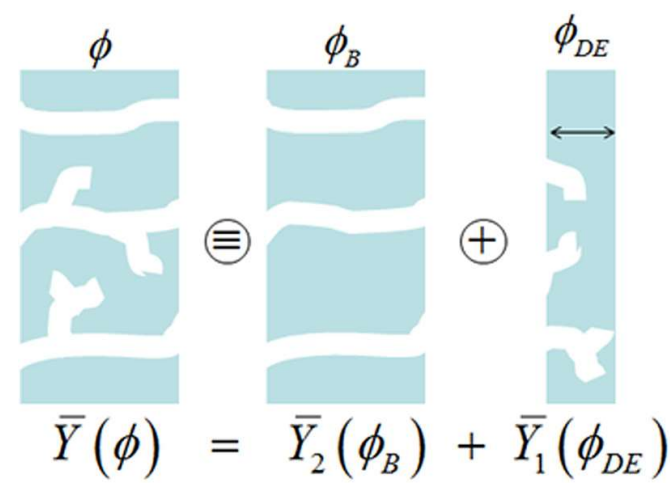

FIG. 5. (Color online) Principle of the procedure to incorporate dead-end porosity effects in any acoustic model. averaged standing wavefield obtained from the contributions of all the dead-ends in the material. If the additional assumption $k l \ll 1$ is made, Eq. (6) becomes, to the first order:

$$
\bar{Y}_{1} \approx \frac{j k}{Z_{C}} \frac{\iiint_{V_{\mathrm{DE}}} l d V}{\iiint_{V_{\mathrm{DE}}} d V} .
$$

This assumption is valid for dead-end pores that are much shorter than any acoustic wavelength. By introducing the average length of the dead-end pores:

$$
l_{\mathrm{DE}}=\frac{\iiint_{V_{\mathrm{DE}}} l d V}{\iiint_{V_{\mathrm{DE}}} d V} .
$$

The admittance (6) and (7) is finally expressed as

$$
\bar{Y}_{1} \approx \frac{j k l_{\mathrm{DE}}}{Z_{C}} .
$$

\section{Cavities involving the dead-end porosity $\phi_{\mathrm{DE}}$ and an effective length of the dead-end pores $I_{\mathrm{DE}}^{e}$}

In the expression of $l_{\mathrm{DE}}$ in Eq. (8), the integration is performed in a homogenization volume $V_{\mathrm{DE}}$ corresponding to the sum of a great number of dead-end pores, so that the length is averaged. It is thought that in full poroelastic problems, an averaged effective length of the dead end pores $l_{\mathrm{DE}}^{e}$ could be used:

$$
l_{\mathrm{DE}}^{e}=\frac{\iiint_{V} l d V}{\iiint_{V} d V} .
$$

The difference with the previous parameter is that the integration is performed in a homogenization volume $V$ of porous aggregate (fluid + solid). This volume should contain enough dead-ends for the average to be valid. The relationship with $l_{\mathrm{DE}}$ is provided by developing the numerator

$$
l_{\mathrm{DE}}^{e}=\frac{\iiint_{V} l d V}{\iiint_{V} d V}=\frac{\iiint_{V_{\mathrm{DE}}} l d V+\iiint_{V_{B}+V_{S}} l d V}{\iiint_{V} d V},
$$

in which the total homogenization volume $V$ is the sum of three terms:

$$
V=V_{\mathrm{DE}}+V_{B}+V_{S}
$$

$V_{\mathrm{DE}}$ corresponds to the volume occupied by the fluid in the dead-ends, $V_{B}$ to the volume occupied by the fluid in the 
kinematic porosity and $V_{S}$ to the volume occupied by the solid inside $V$. The contribution $\iiint_{V_{B}+V_{S}} l d V$ is naught since there is no dead-end inside $V_{B}$ or $V_{S}$. Therefore:

$$
l_{\mathrm{DE}}^{e}=\frac{\iiint_{V_{\mathrm{DE}}} l d V}{\iiint_{V} d V} .
$$

Since

$$
\frac{\iiint_{V_{\mathrm{DE}}} l d V}{\iiint_{V} d V}=\frac{\iiint_{V_{\mathrm{DE}}} l d V}{\iiint_{V_{\mathrm{DE}}} d V} \cdot \frac{\iiint_{V_{\mathrm{DE}}} d V}{\iiint \int_{V} d V}
$$

it is easily seen that the relationship between the lengths $l_{\mathrm{DE}}$ and $l_{\mathrm{DE}}^{e}$ is

$$
l_{\mathrm{DE}}^{e}=\phi_{\mathrm{DE}} l_{\mathrm{DE}}
$$

In Ref. 1 , the validity of the expression for $l_{\mathrm{DE}}$ was demonstrated in the case of the simple geometry of Fig. 6 showing a porous material with surface dead-end pores. Using the same method for $l_{\mathrm{DE}}^{e}$, Eq. (15) is easily retrieved:

$$
\begin{aligned}
l_{\mathrm{DE}}^{e} & =\frac{\iiint_{V} l d V}{\iiint_{V} d V}=\frac{\iiint_{V_{\mathrm{DE}}} l d V}{\iiint_{V} d V}=\underbrace{\frac{V_{\mathrm{DE}} d+V_{\mathrm{DE}} d+\cdots+V_{\mathrm{DE}} d}{V+V+\cdots+V}}_{n} \\
= & \frac{n V_{\mathrm{DE}} d}{n V}=\phi_{\mathrm{DE}} d .
\end{aligned}
$$

The parameter $l_{\mathrm{DE}}^{e}$ should be used in combination with an admittance defined as (in a volume $V$ of porous aggregate):

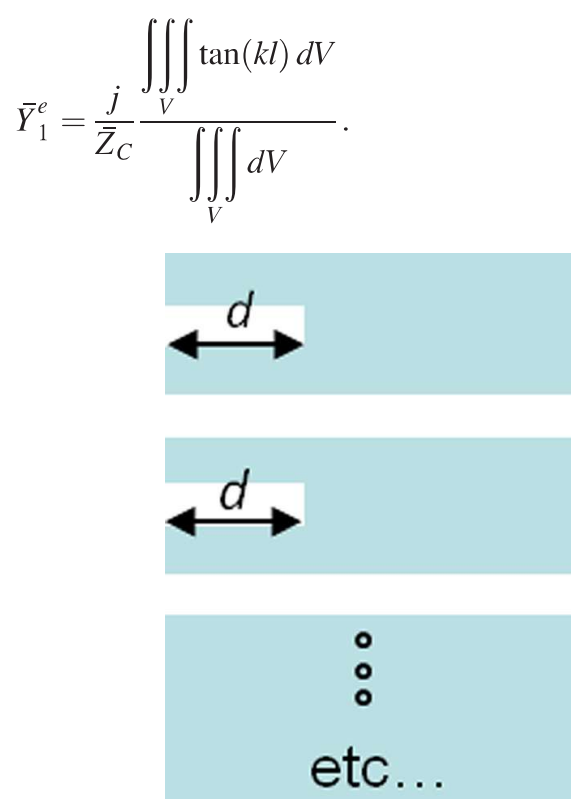

FIG. 6. (Color online) A simple geometry porous material with dead-end porosity.

\section{Possible models for complex geometries}

In the previous models, the dead-end pore is assumed to be of constant cross section and perpendicular to the closing wall. To account for more complicated pore geometries involving Helmholtz resonator geometries for example, or the geometry of the pores of Fig. 1, one should study numerically the acoustic fields in each the dead-ends and try to deduce macroscopic behaviors.

A simpler approach to model complex geometries is to consider that each dead-end pore can be isolated as illustrated in Fig. 5 and treated separately. The problem is then reduced to the calculation of the surface admittance of a layer of thickness $l_{\mathrm{DE}}$ (or $l_{\mathrm{DE}}^{e} / \phi_{\mathrm{DE}}$ ) and backed by a rigid wall. Therefore, the chosen acoustic classical model can be used twice: a first time for the material without dead-end and of porosity $\phi_{B}$ and a second time for a layer of thickness $l_{\mathrm{DE}}$ (or $l_{\mathrm{DE}}^{e} / \phi_{\mathrm{DE}}$ ) and of porosity $\phi_{\mathrm{DE}}$ backed by a rigid wall:

$$
\bar{Y}(\phi)=\bar{Y}_{2}\left(\phi_{B}\right)+\bar{Y}_{1}\left(\phi_{\mathrm{DE}}\right)
$$

In the first and second use of the classical model, the effective density of the fluid and its compressibility should have the same expression. This layer is studied at the macroscopic scale of the laboratory using the classical models. If for example we choose the classical JCA model, the complexity of the microstructure is taken into account through the use in the model of measurable macroscopic parameters (porosity, flow resistivity, tortuosity, characteristic lengths,...).

The approach proposed by Dupont et al. ${ }^{1}$ for dead-end pores was successfully used to model complex materials. It was successfully used to model complex materials that could not be described accurately by classical models. It is based on an assumption that the effect of the dead-end pores can be treated separately (Fig. 5). However, a more fundamental study of the homogenization process will be necessary. It is also thought that the rigorous description of these materials requires the use of general principles in statistical physics such as the ergodicity principle. These studies are ongoing. For the time being, a simple approach was proposed and provided excellent results. It is believed that the model used remains valid if the propagation remains linear (in this case, many behaviors may simply be added up).

\section{E. Description of the dead-end porosity effect with the transfer matrix method (TMM)}

The transfer matrix method (TMM) is a powerful and efficient tool for optimizing and predicting sound absorption and sound transmission of single layer and multilayer sound absorbing materials. If the porous medium with dead-end porosity can be treated as a rigid double layer system (Fig. 5), the TMM is very well adapted to study partially opened porosity materials. The separation of the effects is possible if Eqs. (4) and (18) are valid, i.e., when the Biot and the dead-end contributions in the total admittance can simply be added. The validity of these equations has been discussed in Sec. IV B. The description of dead- 
end porosity materials using the TMM can be formulated as follows:

A vertically periodic unit cell of a non-symmetric porous medium with DE porosity is shown in Fig. 7, where the unit cell separates two fluid domains. The cell is divided into two porous elements in parallel, one with a kinematic porosity and one with a dead-end pore. This approach is slightly different from that of Fig. 5. However, it also provides valid results since the Biot and dead-end contributions can be added. The advantage of considering a unit cell is that the homogenization process can be obtained from considering a collection of these unit cells. This approach is also well adapted to numerical modeling of porous media with for example the finite element method.

The relationship between the acoustic pressures and velocities on both sides of the unit cell is (Dupont et al. ${ }^{1}$ and Panneton et al. $^{45}$ )

$$
\left\{\begin{array}{l}
P \\
U
\end{array}\right\}=[T]^{N S}\left\{\begin{array}{c}
P_{b}^{\prime} \\
-U_{b}^{\prime}
\end{array}\right\}
$$

with

$$
\begin{aligned}
& {[T]^{N S}=-t_{12}^{b}\left[\begin{array}{c}
-\frac{t_{11}^{d e}}{t_{12}^{d e}} \\
\left(\frac{1}{t_{12}^{b}}\right)^{2}-\frac{t_{11}^{b}}{t_{12}^{b}}\left(\frac{t_{22}^{b}}{t_{12}^{b}}+\frac{t_{22}^{d e}}{t_{12}^{d e}}-\left(\frac{1}{t_{12}^{d e}}\right)^{2} \frac{t_{12}^{d e}}{t_{11}^{d e}}\right)
\end{array}\right.} \\
& {\left[\begin{array}{ll}
t_{11}^{b} & t_{12}^{b} \\
t_{21}^{b} & t_{22}^{b}
\end{array}\right]=\left[\begin{array}{cc}
\cos \left(k_{B} l\right) & j Z_{B} \sin \left(k_{B} l\right) \\
\frac{j}{Z_{B}} \sin \left(k_{B} l\right) & \cos \left(k_{B} l\right)
\end{array}\right]} \\
& {\left[\begin{array}{ll}
t_{11}^{d e} & t_{12}^{d e} \\
t_{21}^{d e} & t_{22}^{d e}
\end{array}\right]=\left[\begin{array}{cc}
\cos \left(k_{\mathrm{DE}} l_{\mathrm{DE}}\right) & j Z_{\mathrm{DE}} \sin \left(k_{\mathrm{DE}} l_{\mathrm{DE}}\right) \\
\frac{j}{Z_{\mathrm{DE}}} \sin \left(k_{\mathrm{DE}} l_{\mathrm{DE}}\right) & \cos \left(k_{\mathrm{DE}} l_{\mathrm{DE}}\right)
\end{array}\right]}
\end{aligned}
$$

In the expressions for the elements of the matrices, $k_{B}$ and $Z_{B}$ correspond, respectively, to the wavenumber and the equivalent characteristic impedance relative to the kinematic porosity domain and $l$ represents the thickness of the kinematic porosity element. $k_{\mathrm{DE}}, Z_{\mathrm{DE}}$, and $l_{\mathrm{DE}}$ correspond to their counterparts for the DE element. Assuming the material is backed by an air cavity, the global transfer matrix of the system is given by

$$
[T]^{\text {syst }}=[T]^{N S}[T]^{\mathrm{cav}}
$$

with $[T]^{\text {cav }}$ the transfer matrix of the air cavity. If an air cavity of thickness $l_{\text {cav }}$ is present,

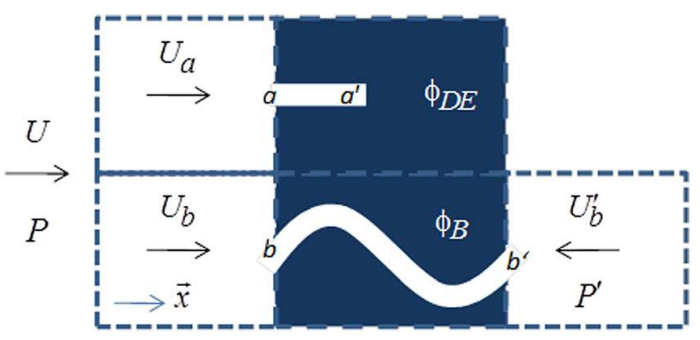

FIG. 7. (Color online) Principle of the model including dead-end elements. $\left.-\left(\frac{t_{22}^{b}}{t_{12}^{b}}+\frac{t_{22}^{d e}}{t_{12}^{d e}}-\left(\frac{1}{t_{12}^{d e}}\right)^{2} \frac{t_{12}^{d e}}{t_{11}^{d e}}\right)\right]$

$$
[T]^{\text {cav }}=\left[\begin{array}{cc}
\cos \left(k_{0} l_{\text {cav }}\right) & j Z_{0} \sin \left(k_{0} l_{\text {cav }}\right) \\
\frac{j}{Z_{0}} \sin \left(k_{0} l_{\text {cav }}\right) & \cos \left(k_{0} l_{\text {cav }}\right)
\end{array}\right]
$$

with $Z_{0}$ and $k_{0}$, respectively, the characteristic impedance and the wavenumber of air, or if the layer is glued on a rigid backing, $[T]^{\text {cav }} \equiv 1$. Using the coefficients of the global transfer matrix, the useful acoustic indicators are for the transmission loss:

$$
T L=20 \log _{10}\left(\frac{1}{2}\left|T_{11}+T_{22}+\frac{T_{12}}{Z_{0}}+Z_{0} T_{21}\right|\right)
$$

and for the normal incidence absorption coefficient:

$$
\alpha=1-\left|\frac{T_{11}-T_{21} Z_{0}}{T_{11}+T_{21} Z_{0}}\right|^{2} .
$$

An alternative formulation involving a symmetric deadend element is possible (see Refs. 1 and 45). This other approach is best suited to the study of systems with symmetric features such as materials with surface dead-ends located on the backside of the sample.

It may be noticed that the TMM approach proposed in this section was used recently to characterize the acoustic properties of locally reacting patches (Panneton et $\left.a l .^{46}\right)$.

\section{RESULTS ON THE MODEL IMPLEMENTATION AND ON THE EXPERIMENTAL VALIDATION}

Experiments were conducted on various materials likely to contain dead-end pores such as metallic foams (Fig. 1) or specially fabricated to incorporate them. An example of specially designed material with well-controlled parameters ("simplified sample") is shown in Fig. 8. Results, mainly 


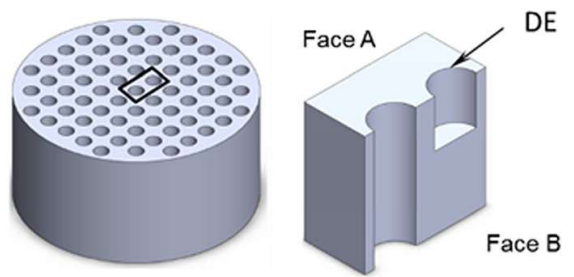

FIG. 8. (Color online) Simplified sample with well-controlled parameters specially fabricated to incorporate dead-end porosity [Panneton et al. (Ref. 45)].

obtained in Ref. 1, are shown in this section to demonstrate the validity of the models for dead-end porosity. More details on the experimental procedure, on the sample specifications, and on the material properties can be found in this reference. The material properties are summarized in Table I. Figures 9-11 clearly show that the classical JCA model cannot describe very well the acoustic indicators of the studied materials (Fig. 8) while the transfer matrix method with dead-end provides a much better theoretical/experimental agreement.

\section{RECENT DEVELOPMENTS IN MATERIAL CHARACTERIZATION FOR MATERIALS WITH DEAD-END POROSITY}

Research on the measurement of the dead-end porosity and of the average length of the dead-ends is currently ongoing. First results were obtained recently, some of which were presented at a conference. ${ }^{47}$ At this point, it should be noticed that the dead-end pores in a material may probably have an influence on the other parameters (tortuosity, flow resistivity, characteristic lengths,...). Recent and still ongoing work conducted by the authors seems to show that the presence of dead-end pores significantly influences the tortuosity and, to a lesser extent, the thermal characteristic length and the kinematic porosity. This influence is more pronounced as the ratio of the kinematic and dead-end pores diameters is close to 1 . The effect of lateral cavities on the tortuosity was previously observed by Chevillote et $a l^{22}$ This influence will have to be addressed more thoroughly in future research.

\section{A. Dead-end porosity measurement}

Recent results on new methods for measuring the kinematic porosity (or Biot porosity $\phi_{B}$ ) are now presented. These new methods, associated with the total porosity provides the dead-end porosity. For the measurement of $\phi_{B}$, two methods can be used depending on the data at our disposal, a low and a high frequency methods. The applicability ranges of these methods are different and depends on a parameter $\beta$ defined in Ref. 48. In fact, these two methods are

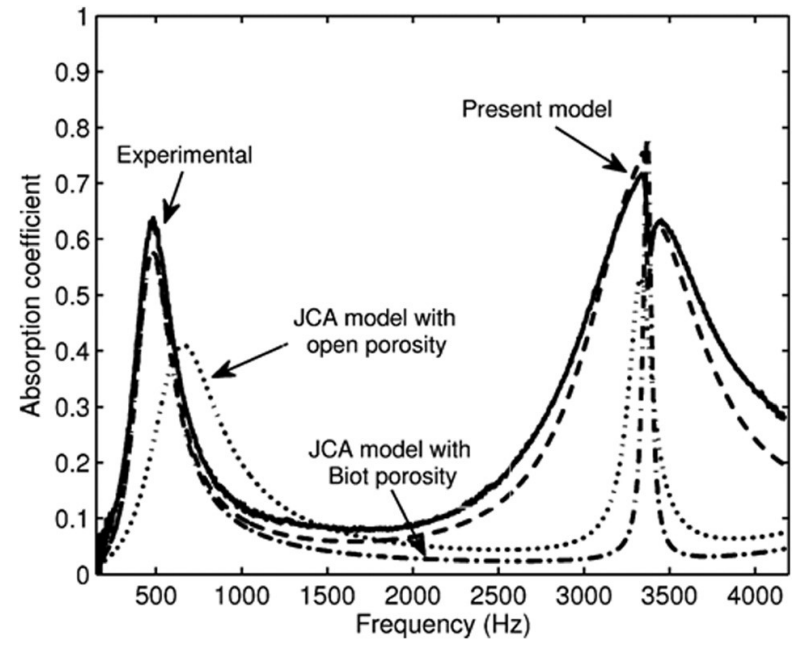

FIG. 9. Comparison between experimental and theoretical results on the absorption coefficient for the simplified materials of Fig. 8. The dotted curve represents the classical JCA model results without accounting for dead-end effects. The dash-dot curve represents the classical JCA model with solely a porosity correction (the kinematic porosity was used instead of the total open porosity).

complementary. The results presented here are taken from Refs. 47 and 48. Only the principles of the methods are presented.

\section{High frequency method}

In this method, high frequency measurements obtained from ultrasonic experiments are used in conjunction with the theoretical value of the intercept with the vertical axis of the modulus of the logarithm of the transmission coefficient. The experimental principle consists in using ultrasonic wave transmission experiments (see Refs. 35 and 36). It can be shown that methods based on ultrasonic reflection cannot be applied here.

In transmission experiments, the porosity actually "seen" by the ultrasonic wave corresponds to the kinematic porosity and therefore provides a measure of $\phi_{B}$. Since, the total open porosity can be measured by classical methods (see Refs. 24-29 or even calculated in the example of Fig. 8), the dead-end porosity can be deduced from these measurements.

Figure 12 illustrates the principle of the method involving the high frequency ultrasonic experimental data, the linear behavior, and the intercept with the vertical axis. The intercept with the vertical axis is obtained by extending the straight line that fits the experimental data. Its value being a

TABLE I. Material properties. For the simplified material, the values are theoretical. For the metallic foam, the parameters were measured experimentally or fitted (for $l_{\mathrm{DE}}$ ).

\begin{tabular}{|c|c|c|c|c|c|c|c|c|}
\hline JCA parameters & $\begin{array}{c}\Lambda \\
(\mu \mathrm{m})\end{array}$ & $\begin{array}{c}\Lambda^{\prime} \\
(\mu \mathrm{m})\end{array}$ & $\alpha_{\infty}$ & $\begin{array}{c}\sigma \\
\left(\mathrm{Pa} \mathrm{s} / \mathrm{m}^{2}\right)\end{array}$ & $\begin{array}{l}\phi_{O} \\
(\%)\end{array}$ & $\begin{array}{l}\phi_{B} \\
(\%)\end{array}$ & $\begin{array}{r}\phi_{\mathrm{DE}} \\
(\%)\end{array}$ & $\begin{array}{c}l_{\mathrm{DE}} \\
(\mathrm{mm})\end{array}$ \\
\hline Simplified material & 900 & 1000 & 1 & 1050 & 27.5 & 14 & 13.5 & 25 \\
\hline Metallic foam & $101 \pm 4$ & $352 \pm 14$ & $2.25 \pm 0.05$ & $19713 \pm 300$ & $64.5 \pm 3$ & $55 \pm 3.2$ & $7.5 \pm 3.2$ & $\approx 7 d_{\text {cell }}$ fitting \\
\hline
\end{tabular}




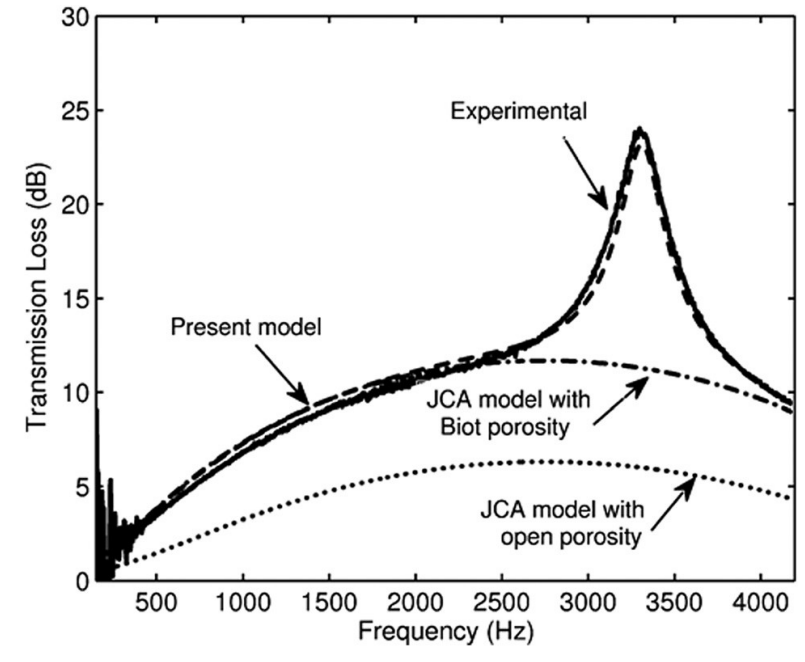

FIG. 10. Comparison between experimental and theoretical results on the transmission loss for the simplified materials of Fig. 8. The dotted curve represents the classical JCA model results without accounting for the dead-end effects. The dash-dot curve represents the classical JCA model with solely a porosity correction (the kinematic porosity was used instead of the total open porosity).

function of porosity (the kinematic porosity here), the determination of the intercept yields the sought porosity.

\section{Low frequency method}

In this method, low frequency measurements obtained from transmission experiments are used in conjunction with the theoretical high frequency asymptotic behavior of the phase velocity of the ultrasonic waves. Figure 13 shows a $\log -\log$ plot of the phase velocity as a function of frequency. It can be seen that the low frequency behavior of the phase velocity can be fitted by a straight line obtained from the experimental data while the JCA model is purely theoretical.

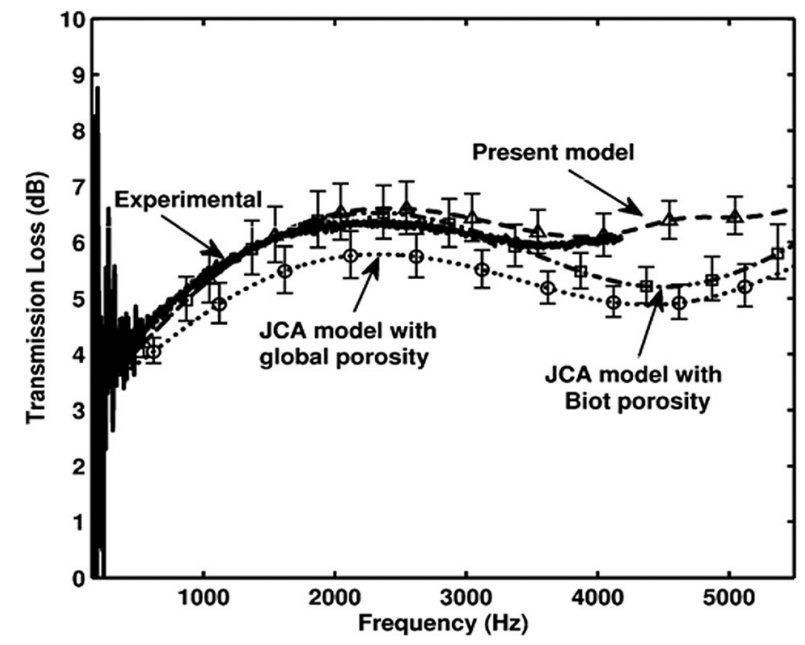

FIG. 11. Comparison between experimental and theoretical results on the transmission loss for the materials of Fig. 1 (see Ref. 1 for details). The dashed line with triangle represents the present model results (symmetric approach), the dash-dot curve with squares represents the classical JCA model with solely a porosity correction (the kinematic porosity was used instead of the total open porosity) and the dotted curve represents the classical JCA model results without accounting for dead-end effects.

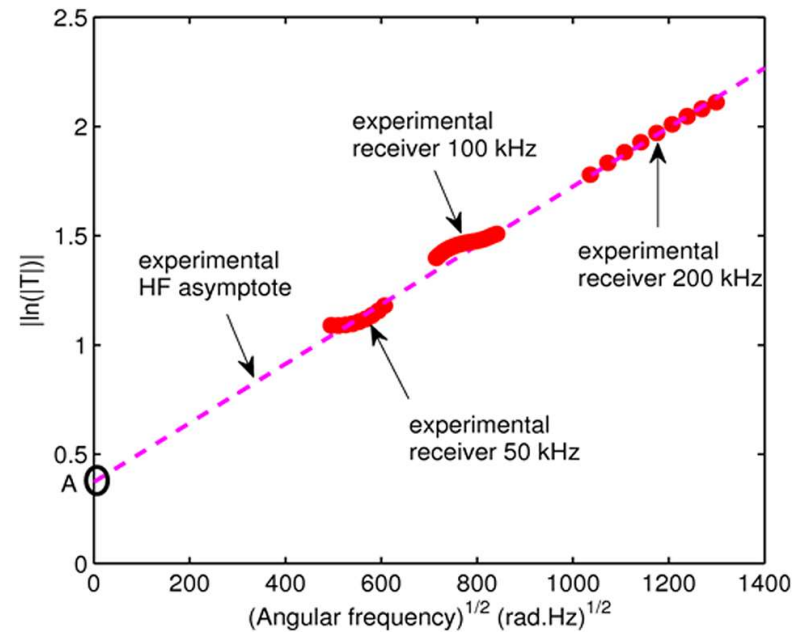

FIG. 12. (Color online) Modulus of the transmission loss (theoretical) and ultrasonic experimental data (experimental) on this sample (Ref. 46). The intercept with the vertical axis provides the kinematic porosity.

This can explain the small discrepancy observed between the straight line and the JCA model at low frequencies. This discrepancy can therefore be attributed to experimental error (in practice, only the straight line will be used). The intercept of the fitted straight line with the theoretical high frequency horizontal asymptote corresponds to the Biot characteristic cutoff frequency separating the low and high frequency behaviors. This frequency is

$$
f_{c}=\sigma \frac{\phi_{B}}{2 \pi \alpha_{\infty} \rho_{f}}
$$

where $\sigma$ is the flow resistivity, $\alpha_{\infty}$ the tortuosity, and $\rho_{f}$ the density of the fluid. These parameters are of course supposed to be known. The Biot frequency depends on the sought porosity (the kinematic porosity here).

Both the low and high frequency methods proposed here are original inasmuch as they involve experimental data

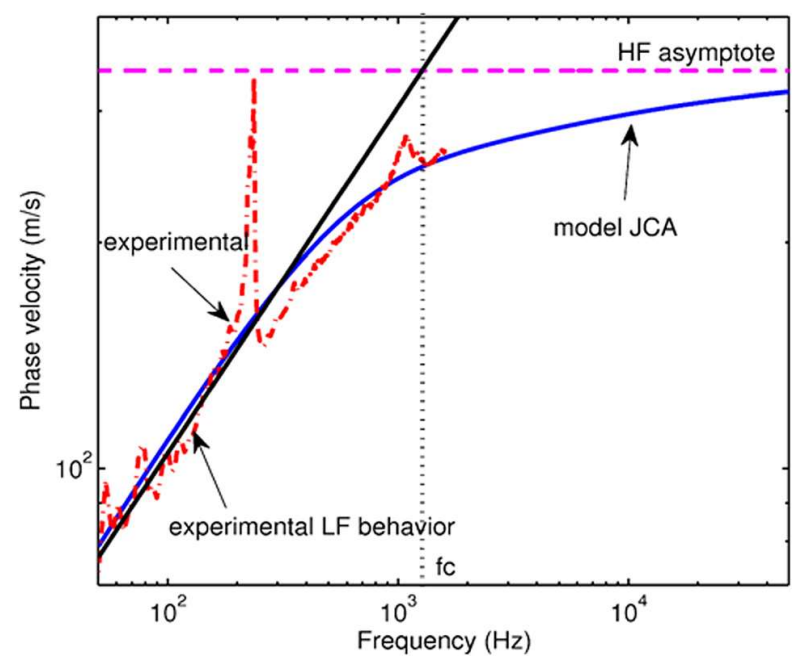

FIG. 13. (Color online) Experimental phase velocity versus frequency, with the experimental low frequency (LF) behavior and the high frequency (HF) asymptote for a melamine foam. The kinematic porosity is provided by the intercept with the horizontal HF asymptote (Ref. 48). 
obtained either from ultrasonic experiments or from experimental data obtained at audible frequencies (transmission tube for example), in combination with low or high frequency asymptotic behavior that are fairly simple.

\section{B. Measurement of the average length of the dead-ends}

The research on methods for the determination of this parameter is currently ongoing. One of the methods currently investigated is the use of the frequency of the resonance due to the dead-end effect in the transmission loss curve. This frequency is directly related to the average length of the dead-ends.

\section{CONCLUSION}

In this article, a description of dead-end porosity systems and their numerous potential applications such as in material recycling or even metamaterial design was proposed in the general context of the study of porous materials, the classical model describing them and the characterization techniques.

It was shown that the dead-end effect can be treated independently and, as a consequence, the dead-end effect can be studied theoretically in combination with any acoustic models. Various models for the description of the dead-end effect were proposed. In this process, a new parameter was introduced, namely the average effective length of the dead-ends.

Recent developments on experimental methods for measuring the parameters of the dead-end porosity materials were also presented. Together with the new theoretical research on dead-end porosity materials, the introduction of these new parameters opens up numerous interesting research scopes in the field of material characterization.

\section{ACKNOWLEDGMENT}

The authors are grateful to Dr. O. Sicot and Professor $X$. L. Gong for their help in the design and fabrication of aluminum foams and for sharing their knowledge on the mechanical properties of these materials.

${ }^{1}$ T. Dupont, P. Leclaire, O. Sicot, X. L. Gong, and R. Panneton, "Acoustic properties of air-saturated porous materials containing dead-end porosity," J. Appl. Phys. 110, 094903 (2011)

${ }^{2}$ J. F. Allard and N. Atalla, Propagation of Sound in Porous Media. Modelling Sound Absorbing Materials (Elsevier, New York, 1993, 1st ed., and Wiley, New York, 2009, 2nd ed.), $372 \mathrm{pp}$.

${ }^{3} \mathrm{G}$. Kirchhoff, "On the influence of heat conduction in a on sound propagation," Ann. Phys. Chem. 134, 177-193 (1868)

${ }^{4}$ I. B. Crandall, Theory of Vibrating Systems and Sound (Van Nostrand, New York, 1927)

${ }^{5}$ C. Zwikker and C. W. Kosten, Sound Absorbing Materials (Elsevier, New York, 1949), pp. 20, 21

${ }^{6} \mathrm{M}$. A. Biot, "Theory of propagation of elastic waves in a fluid-saturated porous solid. I. Low frequency range. II. Higher frequency range," J. Acoust. Soc. Am. 28, 168-191 (1956).

${ }^{7}$ M. E. Delany and E. N. Bazley, "Acoustical properties of fibrous absorbent materials," Appl. Acoust. 3, 105-116 (1970).

${ }^{8} \mathrm{D}$. K. Wilson, "Relaxation-matched modeling of propagation through porous media, including fractal pore structure," J. Acoust. Soc. Am. 94, 1136-1145 (1993).
${ }^{9} \mathrm{~K}$. Attenborough, "Acoustical characteristics of porous materials," Phys. Rep. 82, 179-227 (1982).

${ }^{10}$ D. L. Johnson, J. Koplik, and R. Dashen, "Theory of dynamic permeability and tortuosity in fluid-saturated porous media," J. Fluid. Mech. 176, 379-402 (1987).

${ }^{11}$ Y. Champoux and J. F. Allard, "Dynamic tortuosity and bulk modulus in air-saturated porous media,” J. Appl. Phys. 70, 1975-1979 (1991).

${ }^{12}$ D. Lafarge, P. Lemarinier, J. F. Allard, and V. Tarnow, "Dynamic compressibility of air in porous structures at audible frequencies," J. Acoust. Soc. Am. 102, 1995-2006 (1997).

${ }^{13}$ S. R. Pride, F. D. Morgan, and F. A. Gangi, "Drag forces of porousmedium acoustics," Phys. Rev. B 47, 49644978 (1993).

${ }^{14}$ K. V. Horoshenkov and M. J. Swift, "The acoustic properties of granular materials with pore size distribution close to log-normal," J. Acoust. Soc. Am. 5, 2371-2378 (2001).

${ }^{15} \mathrm{R}$. Panneton, "Comments on the limp frame equivalent fluid model for porous media,” J. Acoust. Soc. Am. 122, EL217-222 (2007).

${ }^{16} \mathrm{~N}$. Atalla, R. Panneton, and P. Debergue, "A mixed displacement pressure formulation for poroelastic materials," J. Acoust. Soc. Am. 104, 1444-1452 (1998).

${ }^{17} \mathrm{P}$. Leclaire, L. Kelders, W. Lauriks, J. F. Allard, and C. Glorieux, "Ultrasonic wave propagation in reticulated foams saturated by different gases: High frequency limit of the classical models," Appl. Phys. Lett. 69, 2641-2643 (1996)

${ }^{18} \mathrm{X}$. Olny and C. Boutin, "Acoustic wave propagation in double porosity media,” J. Acoust Soc. Am. 114, 73-89 (2003).

${ }^{19}$ Z. E. A. Fellah and C. Depollier, "Transient acoustic wave propagation in rigid porous media: A time-domain approach," J. Acoust. Soc. Am. 107, 683-688 (2000).

${ }^{20}$ L. De Ryck, W. Lauriks, P. Leclaire, J. P. Groby, A. Wirgin, and C. Depollier, "Reconstruction of material properties profiles in onedimensional macroscopically inhomogeneous rigid frame porous media in the frequency domain," J. Acoust. Soc. Am. 124, 1591-1606 (2008), and references therein.

${ }^{21}$ G. Gautier, L. Kelders, J. P. Groby, O. Dazel, L. De Ryck, and P. Leclaire, "Propagation of acoustic waves in a one-dimensional macroscopically inhomogeneous poroelastic material," J. Acoust. Soc. Am. 130, 1390-1398 (2011).

${ }^{22}$ F. Chevillotte, C. Perrot, and R. Panneton, "Microstructure based model for sound absorption predictions of perforated closed-cell metallic foams," J. Acoust. Soc. Am. 128, 1766-1776 (2010).

${ }^{23}$ P. Leclaire, T. Dupont, O. Sicot, and X. L. Gong, "Propriétés acoustiques de matériaux poreux saturés d'air incluant une porosité partiellement ouverte" ("Acoustic properties of air-saturated porous materials incorporating a partially opened porosity"), in Proc. 10ème Congrès Français d'Acoustique, CFA, Lyon, France, Apr. 12-16 (2010).

${ }^{24}$ L. L. Beranek, "Acoustic impedance of porous materials," J. Acoust. Soc. Am. 13, 248-260 (1942).

${ }^{25}$ ASTM D2856-94 (1998). Withdrawn Standard: ASTM D2856-94 (1998) "Standard Test Method for Open-Cell Content of Rigid Cellular Plastics by the Air Pycnometer" (withdrawn 2006). Withdrawn 2006 Replaced by ASTM D6226 - 10 "Standard Test Method for Open Cell Content of Rigid Cellular Plastics."

${ }^{26}$ Y. Champoux, M. R. Stinson, and G. A. Daigle, "Air-based system for the measurement of porosity," J. Acoust. Soc. Am. 89, 910-916 (1991).

${ }^{27}$ P. Leclaire, O. Umnova, K. V. Horoshenkov, and L. Maillet, "Porosity measurement by comparison of air volumes," Rev. Sci. Instrum. 74, 1366-1370 (2003).

${ }^{28}$ Y. Salissou and R. Panneton, "Pressure/mass method to measure open porosity of porous solids,” J. Appl. Phys. 101, 124913 (2009).

${ }^{29}$ R. Panneton and E. Gros, "A missing mass method to measure the open porosity of porous solids," Acta Acust. Acust. 91, 342-348 (2005).

${ }^{30}$ ISO 9053:1991, Acoustics-Materials for acoustical applicationsDetermination of airflow resistance (International Organization for Standardization, Geneva, Switzerland, 2001).

${ }^{31} \mathrm{P}$. Lemarinier, M. Henry, J. F. Allard, J. L. Bonardet, and A. Gédéon, "Connection between the dynamic bulk modulus of air in a porous medium and the specific surface," J. Acoust. Soc. Am. 97, 3478-3482 (1995).

${ }^{32}$ D. L. Johnson, T. J. Plona, C. Scala, F. Pasierb, and H. Kojima, "Tortuosity and acoustic slow waves,” Phys. Rev. Lett. 49, 1840-1844 (1982).

${ }^{33} \mathrm{R}$. Panneton and X. Olny, "Acoustical determination of the parameters governing viscous dissipation in porous media," J. Acoust. Soc. Am. 119, 2027-2040 (2006). 
${ }^{34}$ P. B. Nagy, "Slow wave propagation in air-filled permeable solids," J. Acoust. Soc. Am. 93, 3224-3234 (1993).

${ }^{35}$ J. F. Allard, B. Castagnède, M. Henry, and W. Lauriks, "Evaluation of tortuosity in acoustic porous materials saturated by air," Rev. Sci. Instrum. 65, 754-755 (1994).

${ }^{36}$ P. Leclaire, L. Kelders, W. Lauriks, N. R. Brown, M. Melon, and B. Castagnède, "Determination of the viscous and thermal characteristic lengths of plastic foams by ultrasonic measurements in helium and air," J. Appl. Phys. 80, 2009-2012 (1996).

${ }^{37}$ Z. E. A. Fellah, S. Berger, W. Lauriks, C. Depollier, C. Aristegui, and J.-Y. Chapelon, "Measuring the porosity and the tortuosity of porous materials via reflected waves at oblique incidence," J. Acoust. Soc. Am. 113, 2424-2433 (2003).

${ }^{38}$ M. A. Biot and D. G. Willis, "The elastic coefficients of the theory of consolidation,” J. Appl. Mech. 24, 594-601 (1957).

${ }^{39} \mathrm{~T}$. Pritz, "Apparent complex Young's modulus of a longitudinally vibrating viscoelastic rod,” J. Sound Vib. 77, 93-100 (1981).

${ }^{40}$ L. Jaouen, A. Renault, and M. Deverge, "Elastic and damping characterizations of acoustical porous materials: Available experimental methods and applications to a melamine foam," Appl. Acoust. 69, 1129-1140 (2008).

${ }^{41}$ J. F. Allard, G. Jansens, G. Vermeir, and W. Lauriks, "Frame-borne surface waves in air-saturated porous media," J. Acoust. Soc. Am. 111, 690-696 (2002).
${ }^{42}$ L. Boeckx, P. Leclaire, P. Khurana, C. Glorieux, W. Lauriks, and J. F. Allard, "Guided elastic waves in porous materials saturated by air under Lamb conditions,” J. Appl. Phys. 97, 094911 (2005), and references therein.

${ }^{43}$ S. Mahasaranon, K. V. Horoshenkov, A. Khan, and H. Benkreira, "The effect of continuous pore stratification on the acoustic absorption in open cell foams," J. Appl. Phys. 111, 084901 (2012).

${ }^{44}$ D. T. Blackstock, Fundamentals of Physical Acoustics (Wiley, New York, 2000), pp. 156-158.

${ }^{45} \mathrm{R}$. Panneton, T. Dupont, and P. Leclaire, "Modeling of a perforated solid with dead-end porosity by the transfer matrix method," Proc. Symp. Acoustics of Poro-Elastic Materials (SAPEM), Ferrara, Italy (Dec. 14-16, 2011).

${ }^{46}$ R. Panneton, K. Verdière, S. Elkoun, T. Dupont, and P. Leclaire, "Acoustic modeling of locally reacting mosaic materials," in Proc. Internoise 2012, New York (August 19-22, 2012).

${ }^{47}$ T. Dupont, P. Leclaire, and R. Panneton, "Methods for measuring the porosities of porous materials incorporating dead-end pores," Proc. Symp. Acoustics of Poro-Elastic Materials (SAPEM), Ferrara, Italy (Dec. 14-16, 2011).

${ }^{48}$ T. Dupont, P. Leclaire, and R. Panneton, "Acoustic methods for measuring the porosities of porous materials incorporating dead-end pores," J. Acoust. Soc. Am. 133, 2136-2145 (2013). 